\title{
Encoding context effects in recognition and cued recall
}

\author{
VIRGINIA A. DIEHL and DAVID L. HORTON \\ University of Maryland, College Park, Maryland
}

\begin{abstract}
The effect of information activated during encoding on subsequent recognition and cued recall was investigated. Subjects were shown a series of weakly related word pairs and were instructed to remember the word that appeared in capital letters and to look for a relationship between the two words. Four types of distractor words were used in recognition: related to both cue and target, related to target only, related to cue only, and unrelated. The results indicated that correct rejections decreased as relatedness to the target increased. In cued recall, performance decreased as the number of words that were associatively linked to both the cue and target words increased. Both of these outcomes are thought to reflect information activated at the time of encoding.
\end{abstract}

In the encoding specificity paradigm, a series of word pairs is presented during encoding. The to-be-remembered word, or target word, in each pair typically appears in capital letters, and the context word appears in lowercase letters. The instructions to the participants indicate that attending to the context word may assist them in remembering the target word.

In the case of a particular target word, the general context at the time of encoding consists of the instructions as well as the specific context word presented with the target word. Unless the general context is not sufficient for linking the two words, as when the words are particularly difficult to link and the instructions do not provide the necessary assistance (Gardiner \& Tulving, 1980), what is encoded is likely to reflect activated concepts related to the context word, to the target word, and to both the context and target words. Our focus in the present study is with the role that these concepts activated at the time of encoding may play in subsequent recognition and cued recall tasks.

The basic hypothesis regarding recognition in the present experiment was that the ease with which a distractor word could be recognized as "new" would depend on the extent to which it activated target word information. Specifically, distractor words related to both the context and the target words would be the most likely to be called "old"' because of a direct link to the target word as well as a mediated link to the target word through the context word. Distractor words related only to the target words would be called "old" next most often, followed by distractor words related only to context words, and finally by distractor words that were unrelated to either context or target words.

Computing services were provided by the Computer Science Center of the University of Maryland. Requests for reprints should be sent to David L. Horton, Department of Psychology, University of Maryland, College Park, MD 20742.
In cued recall, the focus was on the effectiveness of the context words as retrieval cues for the target words. The hypothesis was that context cue effectiveness would depend on the size of the set of concepts related to both the context word and the target word of a given pair. This is basically a variation on the category set size effect (see Nelson, 1981), and the prediction was that performance in cued recall would vary inversely with the size of the common set of concepts.

\section{METHOD}

\section{Subjects}

Seventy-one undergraduate introductory psychology students participated in this study. The students received extra course credit for their participation.

\section{Materials}

The materials employed in this study were selected from controlled association norms collected by Douglas L. Nelson and his associates (Nelson, personal communication, November, 1983). The word pairs presented during encoding consisted of 30 weakly related pairs (associative strength less than .08 ) for which the common set size could be determined from Nelson's norms. The word pairs chosen had a common set size of one, two, or three words, and there were 10 pairs of words for each common set size. Eight additional pairs, 4 at the beginning and 4 at the end of the list, were included to control for primacy and recency effects. The words in these 8 pairs did not appear in the retention tests.

The recognition task consisted of 130 words. Thirty of these words were the target words from the pairs presented during encoding. The distractor words consisted of 30 words common to both the context word and the target word for each pair, 30 words related only to the target word for each pair, 30 words related only to the context word for each pair, and 10 words unrelated to any context or target word.

\section{Procedure}

The subjects were run in small groups. They studied a series of 38 word pair slides for $5 \mathrm{sec}$ each, and they were instructed to remember the capitalized word (i.e., the target) in each pair and to look for a relationship between the words in each pair. A 10-min numerical problem solving distractor task immediately followed presentation of the pairs.

Following the problem solving task, the subjects were asked to complete the recognition task. They were instructed to identify each of the 
130 words as "old" (i.e., a target) or "new" (i.e., a distractor). After the recognition task was completed, the subjects performed a cued recall task. The context word for each of the 30 critical pairs presented during encoding served as retrieval cues, and the subjects were asked to write down a target word in the space provided next to each context cue.

\section{RESULTS}

The main findings of interest in the recognition task concerned performance on the distractor words. The data, in terms of correct rejections, were converted to proportions so that comparisons could be made between performance on unrelated words and performance on the related word types. The results are shown in Table 1. As is apparent, the results were as hypothesized, and correlated $t$ tests confirmed this observation for each of the planned comparisons [unrelated vs. related to cue only, $t(70)=$ $2.10, p<.05$; related to cue only vs. related to target only, $t(70)=2.24, p<.05$; related to target only vs. related to both, $t(70)=2.71, p<.05$ ].

Performance in cued recall is shown in Table 2. As can be seen, the data indicate that cued recall performance declines as the size of the common set increases. Planned comparisons indicated that common set sizes 2 and 3 differed significantly $[t(70)=2.14, p<.05]$; however, the comparison between common set size 1 and common set size 2 did not reach significance $[t(70)=1.54$, $p>.05]$. In addition to the comparisons reported above, we looked at the number of target words that were correctly recognized as "old" and were also recalled correctly in cued recall as a function of common set size. The means for common set sizes 1,2 , and 3 were 4.66, 4.55 , and 3.97 out of a maximum score of 10 . Planned comparisons indicated that the means for common set sizes 2 and 3 differed significantly $[t(70)=2.38, p<.05]$; however, the comparison between common set sizes 1 and 2 did not reach significance $[t(70)=0.52, p>.05]$.

\section{DISCUSSION}

The findings reported here lead to an analysis of the factors operative during encoding and during the subsequent retention tests. During

Table 1

Proportion of Correct Rejections for Each Distractor Type

\begin{tabular}{lc}
\hline \multicolumn{1}{c}{ Distractor Type } & Proportion Correct Rejection \\
\hline Related to Cue and Target & .812 \\
Related to Target Only & .844 \\
Related to Cue Only & .873 \\
Unrelated to Cue and Target & .909 \\
\hline
\end{tabular}

Table 2

Cued Recall Means and Standard Deviations for Each Common Set Size

\begin{tabular}{|c|c|c|}
\hline Common Set Size & $\boldsymbol{M}$ & $S D$ \\
\hline 1 & 5.45 & 2.22 \\
\hline 2 & 5.08 & 2.12 \\
\hline 3 & 4.52 & 2.50 \\
\hline
\end{tabular}

encoding concepts associated with the target word, the context word, or both words are activated on the basis of some process such as spreading activation (see Collins \& Loftus, 1975). In most cases, concepts associated with both the context and the target words are likely to be activated. These activated concepts become part of the general context at the time of encoding and they may be encoded as well. At the very least, such activated concepts can be said to have been primed.

During the recognition memory test, there is an opportunity for concepts activated during encoding to interfere with memory performance. Distractor words associated with both the target and the context words would be the most likely words to reinstate the encoding context and to mistakenly be labeled as "old" words. Distractor words related only to target words would be next most likely to be called "old," followed by distractor words related only to the context word. This is the exact pattern of findings reported here for recognition memory. Therefore, recognition memory performance on distractor words is interfered with most when the distractor activates most of the information originally encoded. When distractor words activate little (associated to context word only) or none (unrelated words) of this encoded information, recognition memory performance becomes increasingly better.

In the case of cued recall, words or concepts related to both the context and the target words are most likely to have been part of the original encoding environment, whether or not they were also encoded or only primed. In either case, presentation of the context word as a recall cue should tend to again activate such concepts. The more of these concepts that are activated, the poorer cued recall memory performance will be. Again, the findings reported here show precisely that pattern. of results.

As a final point, let us consider recognition memory performance on target words. In comparison with distractor words, target words should most strongly activate the context available at the time of encoding. Presumably, this encoding context includes words activated other than the target word. Such activated concepts differ from the target word only in that they were net part of the physical (sensory) context present at the time of encoding. Therefore, we might well expect memory performance, in terms of percent correct, to be poorer on target words than on distractor words of the type employed in this study. The results show recognition memory performance on target words at $71 \%$ correct and on distractor words in the most difficult condition (word related to both context and target) at $81.2 \%$ correct.

The results of the present investigation indicate that a variety of conceptual material is likely to be activated during the encoding of an event. Furthermore, the findings also suggest that this activated conceptual material plays a role in subsequent recognition and cued recall tasks. In the case of recognition, the greater the degree to which a distractor word activates the information originally encoded, the more likely it is to be incorrectly called "old." The data also suggest that a similar process may explain errors made on target items. In cued recall, it is the number of activated concepts that are common to both the cue and the target words that plays the main role. The greater the number of common concepts, the poorer the performance in cued recall.

\section{REFERENCES}

Collins, A. M., \& LofTus, E. F. (1975). A spreading activation theory of semantic processing. Psychological Review, 82, 407-428.

Gardiner, J. M., \& TUlviNG, E. (1980). Exceptions to recognition failure of recallable words. Journal of Verbal Learning \& Verbal Behavior, 19, 194-209.

Nelson, D. L. (1981). Many are called but few are chosen: The influence of context on the effects of category size. In G. H. Bower (Ed.), The psychology of learning and motivation (Vol. 15, pp. 129163). New York: Academic Press.

(Manuscript received for publication March 14, 1988.) 